\title{
Caracterización de la mineralización del proceso estilohioideo en tomografías computarizadas volumétricas
}

\author{
Characterization of the mineralization of the stylohyoid process in volumetric computed tomography \\ Roger Sifuentes-Alcazaba ${ }^{1, a ; 2, b}$, Alexis Evangelista-Alva ${ }^{1, c}$, Milushka Quezada-Márquez ${ }^{1, b, c}$
}

\begin{abstract}
RESUMEN
Objetivo: Analizar las características tomográficas de la mineralización del proceso estilohioideo (MPE) mediante tomografía computarizada volumétrica (TCV). Material y Métodos: Se revisaron 860 tomografías computarizadas volumétricas (593 corresponden al sexo femenino y 267 al sexo masculino) de un servicio de Radiología Bucal y Maxilofacial. El rango etario estuvo comprendido entre 6 a 89 años. Se utilizó la adecuación tomográfica de la clasificación de Langlais. El análisis estadístico se realizó mediante el uso de la prueba chi-cuadrado. Resultados: Se encontraron 289 MPE, prevaleció la mineralización bilateral (68.86\%). La longitud promedio aritmética de las MPE fue de $39.54 \mathrm{~mm}$. La frecuencia de la MPE fue del $33.60 \%$ (289 casos). Se encontró mayor frecuencia en el grupo etario de 40 a 49 años, no hubo diferencia significativa entre el sexo femenino y masculino $(\mathrm{p}=0.53$ ). En la clasificación de Langlais el tipo más frecuente fue la variante segmentado con 42.2\%. El patrón de MPE más frecuente fue la variantes parcialmente calcificada con 58.8\%. Conclusiones: La frecuencia de la MPE fue de $33.60 \%$ y la TCV permite identificar oportunamente esta variación anatómica.
\end{abstract}

PALABRAS CLAVE: Tomografía computarizada volumétrica, hueso temporal, variación anatómica.

\section{SUMMARY}

Objective: To evaluate the tomographic characteristics of the mineralization of the stylohyoid process by volumetric computed tomography (CT). Material and Methods: reviewed 860 volumetric computed tomographies (593 correspond to the female sex and 267 to the male sex) of the Oral and Maxillofacial Radiology Service. The age range was between 6 to 89 years. The tomographic adequacy of the Langlais classification was used. The statistical analysis was performed using the chi-square test. Results: 289 MPE were found, bilateral mineralization prevailed (68.86\%). The arithmetic average length of the MPE was $39.54 \mathrm{~mm}$. The frequency of MPE was $33.60 \%$ ( 289 cases). It was found more frequently in the age group of 40 to 49 years, there was no significant difference between the female and male sex $(p=0.5367)$. In the classification of Langlais the most frequent type was the segmented variants with $42.2 \%$. The most frequent MPE pattern was the partially calcified variants with $58.8 \%$. Conclusions: The frequency of the MPE was $33.60 \%$ and the TCV allows to identify this anatomical variation in a timely manner.

KEY WORDS: Cone-beam computer tomography, temporal bone, anatomic variation.

Facultad de Estomatología Roberto Beltrán, Universidad Peruana Cayetano Heredia. Lima, Perú.

Facultad de Odontología, Universidad de San Martin de Porres. Lima, Perú.

Cirujano Dentista, ${ }^{\mathrm{b}}$ Especialista en Radiología Oral y Maxilofacial, " Magister en Estomatología 


\section{INTRODUCCIÓN}

El hueso temporal está constituido por la porción petrosa, la escama del temporal y área timpánica; en la cara pósteroinferior de la porción petrosa del temporal se encuentra la apófisis estiloides (AE) que es una extensión o elongación cilíndrica. La longitud puede variar de 20 a $25 \mathrm{~mm}$., (1,2). Monsour considera normal la longitud de hasta $3 \mathrm{~cm}$ en radiografías panorámicas (3).

La apófisis estiloides (AE) da inserción al Ramillete de Riolano, conformado por los ligamentos estilohioideo, estilomandibular y músculos estilofaríngeo estilogloso y estilohioideo $(4,5)$. El ligamento estilohiodeo se inserta en el vértice de la $\mathrm{AE}$ hasta el asta menor del hioides $(2,5,6)$. El conjunto de la apófisis estiloides y el ligamento estilohioideo es considerado como el proceso estilohioideo (PE) $(2,6)$.

Cuando los cristales minerales se distribuyen de forma organizada en la matriz de los seres vivos se denomina proceso de mineralización (7). La mineralización del ligamento estilohioideo se pueden explicar en tres teorías. La primera de ellas es la hiperplasia reactiva en la cual tejido conjuntivo residual se transforma en fibrocartílago y luego se mineraliza. La metaplasia reactiva es considerada una respuesta de reparación ósea post-traumática aberrante y es una segunda teoría. La tercera teoría propone que hay mineralización del ligamento sin la presencia de trauma previo y es conocida como variación antómica (8). Carmada et al. afirman que es posible que se provo- que una reacción inflamatoria localizada debido a la perdida de elasticidad del ligamento estilohiodeo provocando en la interfase entre el ligamento estilohioideo y el cuerno inferior del hueso hioides una tendinitis, los cuales se manifiestan sin existir incluso evidencia radiográfica de mineralización (9).

\section{Clasificación imagenológica del proceso estilohioideo calcificado.}

Langlais et al., determinaron tres tipos de presentación radiográfica (10) (figura 1):

Tipo I o elongado: integridad completa completa del PE.

Tipo II o pseudoarticulado: la AE se encuentra en proximidad al ligamento estilohioideo o al estilomandibular a través de una pseudoarticulación.

Tipo III o segmentado: la MPE se encuentra fragmentada e interrumpida. Se observan dos o más fragmentos.

Langlais et al. También describen cuatro patrones en la mineralización del $\mathrm{PE}^{10}$ (Figura 2):

Contorno calcificado. La MPE se presenta con un borde o silueta corticalizada y en el área central se observa hueso trabecular.

Parcialmente calcificada.- el borde esta corticalizado y elárea central están casi completamente radiopaca.

Nodular.- la silueta externa es ondulada y el área central puede o no estar completamente radiopaca.

Completamente Calcificada.- el PE es radiopaco en el contorno y el área central.
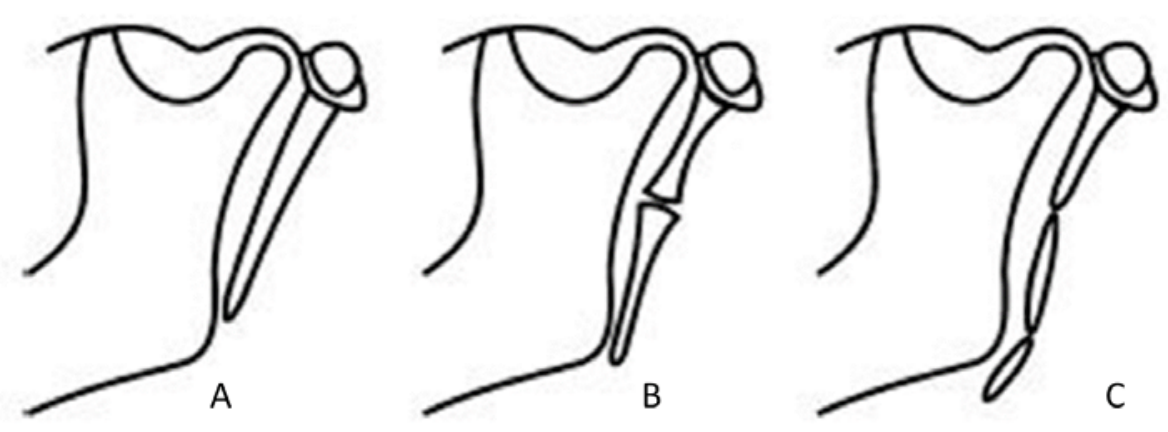

Figura 1. Tipos de la MPE. A: Elongado, B: Pseudoarticulado, C: Segmentado (10). 

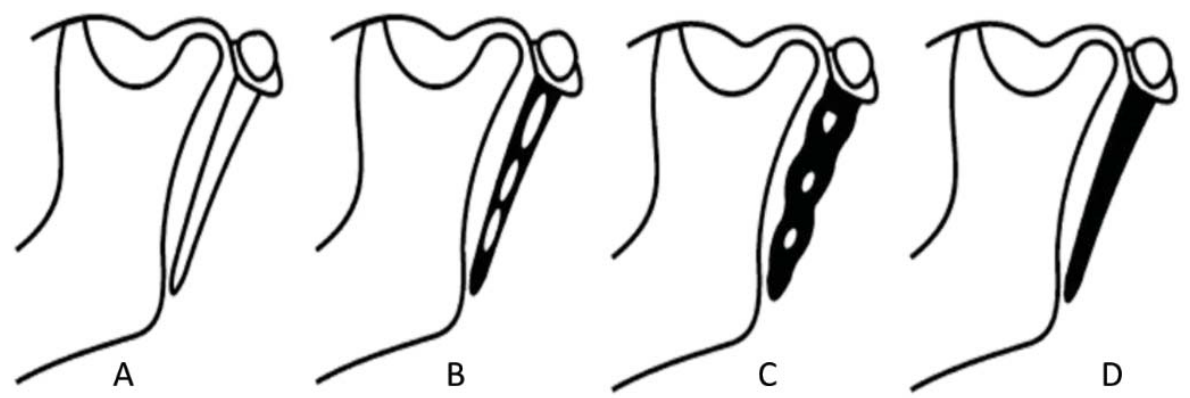

Figura 2. Patrones de la MPE. A: contorno Calcificado, B: Parcialmente calcificada, C: Nodular, D: Completamente Calcificada (10).

\section{Síndrome de Eagle}

Se presenta clínicamente con dolor constante a nivel de la faringe, se agrava al deglutir o pasar los alimentos, con frecuencia el dolor se manifiesta en el oído ipsilateral, puede presentarse nauseas, incremento en la salivación y a veces la impresión de tener un cuerpo extraño en la garganta. Algunos autores indican que el dolor es debido al proceso de cicatrización en el área circundante a la apófisis estiloides luego de amigdalectomía. Además, se propuso una variante, el síndrome estilocarotideo, en el cual la arteria carótida y su proximidad a la apófisis estiloides mineralizada podía causar carotidinia (dolor por compresión de la arteria carótida) y cefaleas intermitentes, mareos y desmayos (6). El objetivo de la investigación fue analizar las características tomográficas de la mineralización del proceso estilohioideo mediante tomografía computarizada volumétrica (TCV).

\section{MATERIAL Y MÉTODOS}

El diseño de este trabajo de investigación fue descriptivo, observacional y transversal. La toma de muestra fue no probabilístico y por conveniencia utilizando 860 tomografías computarizadas volumétricas (TCV) que cumplían con los criterios de inclusión, tomadas en un Servicio de Radiología Bucal y Maxilofacial. Se incluyeron las TCV nítidas de pacientes de ambos sexos, el rango de edad fue de 6 a 89 años y cuya MPE sea igual o mayor a $30 \mathrm{~mm}$. Se excluyeron las TCV de aquellos casos que presentaron patologías quísticas o tumorales del área temporal, traumatis- mos óseos evidentes, malformaciones óseas y TCV con errores de movimiento. Las variables fueron longitud, localización, sexo, tipo y patrón de MPE.

El investigador principal fue preparado y calibrado por un radiólogo bucal y maxilofacial con una experiencia mayor a 10 años. El coeficiente de correlación intraclase en la variable longitud de MPE fue 0.91 intraobservador y 0.88 interobservador. El tipo y patrón de la MPE fue de 0.92 intraobservador y 0.9 interobservador, no hubo diferencia significativa. En la comparación de las variables localización según tipo, patrón de mineralización y sexo se utilizó la prueba estadística de Chi Cuadrado $\left(\mathrm{Ch}^{2}\right)$, los valores se trabajaron con un nivel de significancia de $0.05 \mathrm{y}$ un intervalo de confianza al 95\%.

\section{RESULTADOS}

De las 860 tomografías computarizadas volumétricas, $267(31,05 \%)$ correspondieron al sexo masculino y $593(68,95 \%)$ al sexo femenino. Las MPE encontradas fueron $289(33,60 \%)$ casos. Las medidas de tendencia central se detallan en la tabla 1. El 68,86\% fueron bilaterales y $31,14 \%$ fueron unilaterales siendo $67,78 \%$ del lado derecho y $32,22 \%$ del lado izquierdo. Las frecuencias con respecto al tipo y patrón de MPE según la clasificación adaptada de Langlais se detallan en las tablas 2 y 3 (figura 3 y figura 4 ). 
Tabla 1. Medidas de tendencia central de las MPE según lado.

\begin{tabular}{llccccc}
\hline Bilateralidad & Lado & $\mathrm{n}$ & $\begin{array}{c}\text { Mínimo } \\
(\mathrm{mm})\end{array}$ & $\begin{array}{c}\text { Máximo } \\
(\mathrm{mm})\end{array}$ & $\begin{array}{c}\text { Media } \\
(\mathrm{mm})\end{array}$ & $\begin{array}{c}\text { Desviación } \\
\text { estándar }\end{array}$ \\
\hline \multirow{3}{*}{ Bilateral } & Derecho & 199 & 30,98 & 80,66 & 40,73 & 8,52 \\
& Izquierdo & 199 & 19,34 & 83,01 & 39,45 & 9,07 \\
\multirow{3}{*}{ Unilateral } & Derecho & 61 & 30,22 & 53,04 & 35,99 & 5,01 \\
& Izquierdo & 29 & 31,4 & 67,84 & 39,16 & 8,19 \\
\hline
\end{tabular}

Tabla 2. Distribución de la MPE según tipo y lado.

\begin{tabular}{ccccc}
\hline & & Derecho & \multicolumn{2}{c}{ Izquierdo } \\
Tipo & $\mathrm{n}$ & $\%$ & $\mathrm{n}$ & $\%$ \\
\hline I & 110 & 38,1 & 102 & 35,3 \\
II & 28 & 9,7 & 26 & 9,0 \\
III & 122 & 42,2 & 100 & 34,6 \\
Total & 260 & 90,0 & 228 & 78,9 \\
\hline
\end{tabular}

Tabla 3. Distribución de la MPE según patrón y lado

\begin{tabular}{lcccc}
\hline \multicolumn{1}{c}{ Patrón } & \multicolumn{2}{c}{ Derecho } & \multicolumn{2}{c}{ Izquierdo } \\
& $\mathrm{n}$ & $\%$ & $\mathrm{n}$ & $\%$ \\
\hline Contorno Calcificado & 17 & 5,9 & 15 & 5,2 \\
Parcialmente Calcificado & 170 & 58,8 & 147 & 50,9 \\
Nodular & 73 & 25,3 & 63 & 21,8 \\
Completamente Calcificado & 0 & 0,0 & 3 & 1,0 \\
Total & 260 & 90,0 & 228 & 78,9 \\
\hline
\end{tabular}



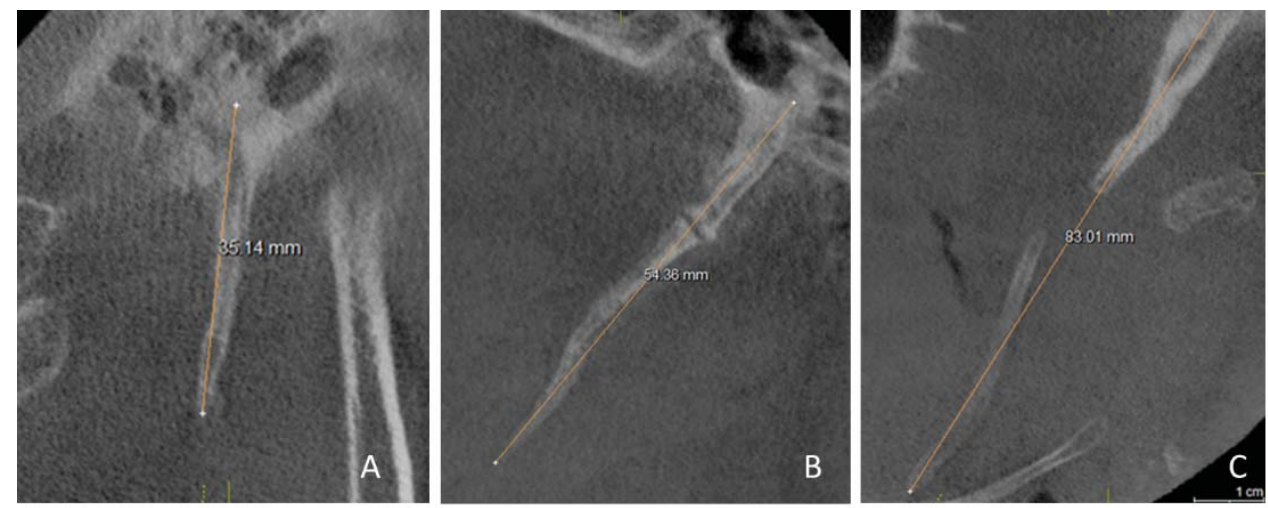

Figura 3. Tipos de la MPE. A: Elongado, B: Pseudoarticulado, C: Segmentado. Tomado del archivo del Servicio de Radiología Bucal y Máxilo facial de la Clínica Dental docente de la Universidad Peruana Cayetano Heredia.
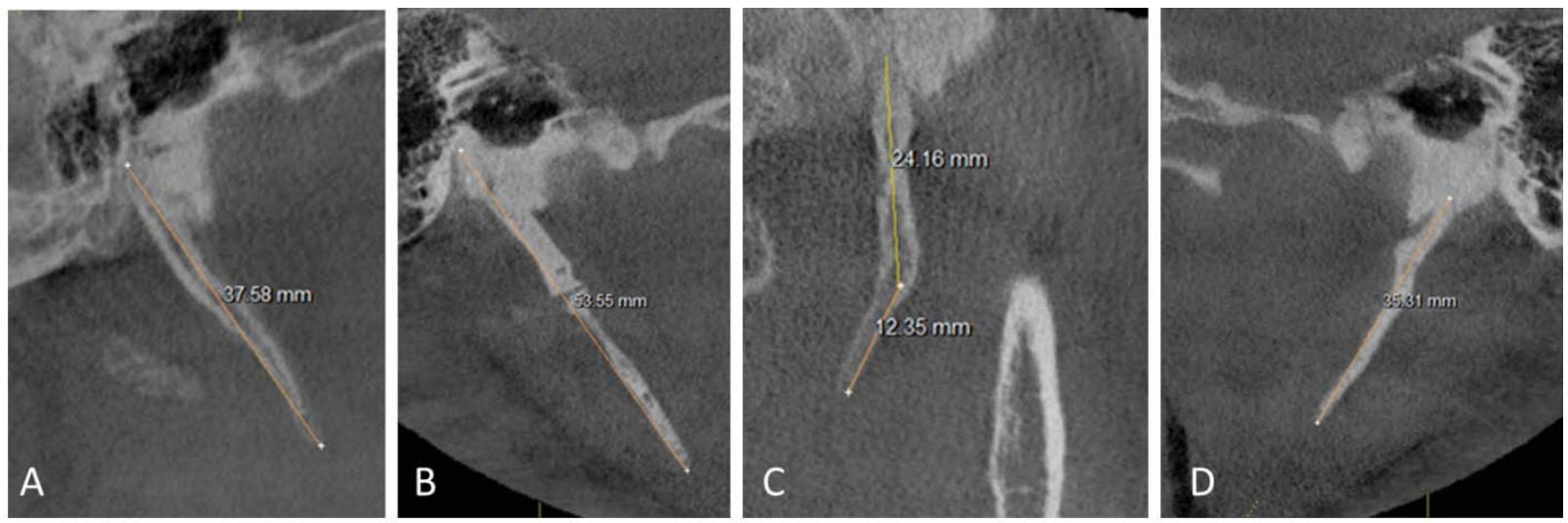

Figura 4. Patrones de la MPE. A: contorno Calcificado, B: Parcialmente calcificada, C: Nodular, D: Completamente Calcificada. Tomado del archivo del Servicio de Radiología Bucal y Máxilo facial de la Clínica Dental docente de la Universidad Peruana Cayetano Heredia. 


\section{DISCUSIÓN}

Cada ser humano es único anatómicamente en tal medida que incluso los gemelos idénticos no son iguales. El término "proceso estiloides" se deriva de la palabra griega "Stylos" que significa un pilar. El proceso estiloides normalmente es un hueso cilíndrico que surge del hueso temporal frente al foramen estilomastoideo. Las estructuras adjuntas incluyen los músculos estilofaríngeos, estilohioides y estiloglosos, y los ligamentos estilohioideos y estilomandibulares (5).

Las mediciones de las longitudes de las MPEs se evaluaron en cortes transaxiales y los casos que midieron más de $30 \mathrm{~mm}$ de longitud fueron tabuladas de acuerdo al lado, sexo, y distribuidas según el tipo y patrón de la clasificación de Langlais (10).

La MPE o la elongación y los cambios estructurales con sus síntomas clínicos han sido descritos por primera vez por Eagle. Por esta razón, estos son conocidos como el síndrome de "Eagle" (6).

Las longitudes máximas observadas en el presente estudio alcanzaron $80.66 \mathrm{~mm}$ en el lado derecho y 83,01 mm en el lado izquierdo. En el año 2015, Khojastepour et al., (11), empleando radiografías panorámicas observaron longitudes en promedio de $39,57 \mathrm{~mm}$ en el lado derecho y $38,31 \mathrm{~mm}$ en el lado izquierdo; Abdel
Ghady et al., en Egipto (12), en un estudio con tomografia espiral multicorte (TEM) encontraron longitud promedio de 43,6 y 43,7 $\mathrm{mm}$ en el lado izquierdo y derecho respectivamente. En Irán en un estudio empleando radiografías panorámicas, reportan longitudes promedio de $37,68 \mathrm{~mm}$ en sexo masculino y $36,32 \mathrm{~mm}$ en femenino (13). Oztunc et al., en Turquía y empleando TCV encuentra longitudes promedio de $36,68 \mathrm{~mm}$ en el sexo masculino y $38,94 \mathrm{~mm}$ en el sexo femenino (14).

El porcentaje de frecuencia de la MPE ha sido reportado entre el $43,7 \%$ y $63,6 \%$. En este estudio la frecuencia fue $33,6 \%$ (289 casos) de un total de $860 \mathrm{TV}$. Oztunc et al., en Turquía encontraron una frecuencia de 63,6\% de MPE de 208 TV evaluadas (14). En Turquia, Basekin et al., encontraron que el 59.4\% de los casos examinados presentaron MPE (15). En Estados Unidos, Akar et al., revelaron un 55\% de MPE en 616 radiografías panorámicas, no sea encontrado relación con respecto al grupo étnico (16)( tabla 4).

El tipo "segmentado" fue el más frecuente de las MPE pero no coincide con Oztunc et al. ${ }^{14}$ quienes también usaron TCV pero concluyeron que el tipo de MPE más frecuente fue el pseudoarticulado; dentro de los estudios realizados con TC tenemos los realizados en Egipto (12) y Turquia (15). En Turquía concluyeron

Tabla 4. Frecuencia de mineralización del proceso estilohiodeo según revisión de la literatura.

\begin{tabular}{|c|c|c|c|c|c|c|}
\hline Autores & Año & País & $\begin{array}{c}\text { Total de } \\
\text { pacientes }\end{array}$ & $\begin{array}{c}\mathbf{N}^{\circ} \text { de } \\
\text { pacientes con } \\
\text { MPE } \\
\end{array}$ & $\%$ & $\begin{array}{c}\text { Técnica } \\
\text { Imagenológica }\end{array}$ \\
\hline Sifuentes & 2017 & Perú & 860 & 289 & $33,60 \%$ & $\mathrm{TCV}$ \\
\hline Akar et al.., ${ }^{16}$ & 2016 & USA & 616 & 339 & $55,00 \%$ & $\begin{array}{l}\text { Radiografía } \\
\text { Panorámica }\end{array}$ \\
\hline Khojastepour et al., ${ }^{11}$ & 2015 & Irán & 350 & 153 & $43,70 \%$ & $\begin{array}{l}\text { Radiografía } \\
\text { Panorámica }\end{array}$ \\
\hline Marcal et al., ${ }^{18}$ & 2015 & Brasil & 736 & 323 & $43,90 \%$ & $\begin{array}{l}\text { Radiografía } \\
\text { Panorámica }\end{array}$ \\
\hline Oztunc et al., ${ }^{14}$ & 2014 & Turquía & 208 & 112 & $63,60 \%$ & $\mathrm{TCV}$ \\
\hline Andrei et al., ${ }^{17}$ & 2013 & Rumanía & 44 & 28 & $63,60 \%$ & $\mathrm{TCV}$ \\
\hline Sudhakara et al., ${ }^{13}$ & 2013 & India & 260 & 154 & $59,20 \%$ & $\begin{array}{l}\text { Radiografía } \\
\text { Panorámica }\end{array}$ \\
\hline Abdel Ghany et al., ${ }^{12}$ & 2013 & Egipto & 18 & 10 & $55,60 \%$ & TEM \\
\hline Basekin et al.,(15) & 2005 & Turquía & 138 & 82 & $59,40 \%$ & TEM \\
\hline
\end{tabular}


que el tipo de MPE más prevalente fue el elongado; en referencia a estudios realizados con radiografías panorámicas citamos a Akar et al.,(16), Marcal et al.,(18), Bagga et al., (19), y Paula et al.,(20), todos estos estudios coincidieron que el tipo elongado de MPE fue el más frecuente, pero que Khojastepour et al., (11), encontraron que el tipo de MPE pseudoarticulado como el más frecuente (tabla 5).
El patrón "parcialmente calcificado" fue el más frecuente en el presente estudio coincidiendo con Oztunc et al. (14) en población turca. Sudhakara et al. ${ }^{13}$ empleando radiografías panorámicas encontraron que el patrón más frecuente fue el calcificado; Bagga et al., (19) en una población Indú observaron que el patrón más frecuente fue el parcialmente calcificado, ste último coincide con nuestro estudio (tabla 6).

Tabla 5. Frecuencia del tipo de mineralización del proceso estilohiodeo según revisión de la literatura.

\begin{tabular}{|c|c|c|c|c|c|}
\hline Autores & Año & País & $\begin{array}{l}\text { Total de } \\
\text { MPE }\end{array}$ & $\begin{array}{l}\text { Tipo de MPE } \\
\text { más frecuente }\end{array}$ & $\begin{array}{c}\text { Técnica } \\
\text { Imagenológica }\end{array}$ \\
\hline Sifuentes & 2017 & Perú & 289 & Segmentado & TCV \\
\hline Akar et al., ${ }^{16}$ & 2016 & USA & 339 & Elongado & $\begin{array}{l}\text { Radiografía } \\
\text { panorámica }\end{array}$ \\
\hline Marcal et al., ${ }^{18}$ & 2015 & Brasil & 323 & Elongado & $\begin{array}{l}\text { Radiografía } \\
\text { panorámica }\end{array}$ \\
\hline Khojastepour et al., ${ }^{11}$ & 2015 & Irán & 153 & Pseudoartic & $\begin{array}{l}\text { Radiografía } \\
\text { Panorámica }\end{array}$ \\
\hline Oztunc et al., ${ }^{14}$ & 2014 & Turquía & 112 & Pseudoartic & $\mathrm{TCV}$ \\
\hline Abdel Ghady et al., ${ }^{12}$ & 2013 & Egipto & 10 & Elongado & TEM \\
\hline Bagga et al.; 19 & 2012 & India & 1411 & Elongado & $\begin{array}{l}\text { Radiografía } \\
\text { Panorámica }\end{array}$ \\
\hline Paula et al., ${ }^{20}$ & 2008 & Brasil & 132 & Elongado & $\begin{array}{l}\text { Radiografía } \\
\text { Panorámica }\end{array}$ \\
\hline Basekin et al., ${ }^{15}$ & 2005 & Turquía & 82 & Elongado & TEM \\
\hline
\end{tabular}


Tabla 6. Frecuencia del patrón de mineralización del proceso estilohiodeo según revisión de la literatura.

\begin{tabular}{|c|c|c|c|c|c|}
\hline Autores & Año & País & $\begin{array}{l}\text { Total de } \\
\text { MPE }\end{array}$ & $\begin{array}{c}\text { Patrón de } \\
\text { MPE más frecuente }\end{array}$ & $\begin{array}{c}\text { Técnica } \\
\text { Imagenológica }\end{array}$ \\
\hline Sifuentes & 2017 & Perú & 289 & $\begin{array}{l}\text { Parcialmente } \\
\text { calcificado. }\end{array}$ & $\mathrm{TCV}$ \\
\hline Oztunc et al., ${ }^{14}$ & 2014 & Turquía & 112 & $\begin{array}{l}\text { Parcialmente } \\
\text { calcificado. }\end{array}$ & $\mathrm{TCV}$ \\
\hline Sudhakara et al., ${ }^{13}$ & 2013 & India & 154 & Calcificado & $\begin{array}{l}\text { Radiografía } \\
\text { Panorámica }\end{array}$ \\
\hline $\begin{array}{l}\text { Bagga et al., } 19 \\
\text { al., }{ }^{19}\end{array}$ & 2012 & India & 1411 & $\begin{array}{l}\text { Parcialmente } \\
\text { calcificado. }\end{array}$ & $\begin{array}{l}\text { Radiografía } \\
\text { Panorámica }\end{array}$ \\
\hline Anbiaee et al. ${ }^{21}$ & 2011 & Irán & 106 & Bordes calcificados & $\begin{array}{l}\text { Radiografía } \\
\text { Panorámica }\end{array}$ \\
\hline
\end{tabular}

\section{CONCLUSIONES}

La MPE puede detectarse fácilmente en tomografías volumétricas. Por lo tanto, puede ayudar a distinguir este problema de otros tipos de mineralización de tejidos blandos y evaluarlo clínicamente, es de ayuda para el diagnóstico de Síndrome de Eagle.

\section{Correspondencia:}

Roger Benito Sifuentes Alcazaba

Correo electrónico: roger.sifuentes.a@upch.pe

\section{REEFERENCIAS BIBLIOGRÁFICAS}

1. Gonzáles E, Aldape B, Luna M, Martín F. Tomografía Cone Beam 3D, Atlas de aplicaciones en odontología. Caracas: AMOLCA; 2014.

2. Bouchet A, Cuilleret J. Anatomía descriptiva, topográfica y funcional: Cara, cabeza y órganos de los sentidos. 21 edición. Buenos Aires: Ed. Medica Panamericana; 1988.

3. Monsour P, Young W. Variability of the styloid process and stylohyoid ligament in panoramic radiographs. Oral Surg Oral Med Oral Pathol. 1986; 61(5): 522-6.

4. Rouvière H, Delmas A, Delmas V. Anatomía descriptiva de la cabeza y del cuello. 11va Edición. Madrid: Elsevier Mason; 2005.

5. Fini G, Gasparini G, Filippini F, Becelli R, Marcotullio D. The long styloid process syndrome or Eagle's syndrome. J Craniomaxillofac Surg. 2000; 28(2):123-7.

6. Raffo M. Síndrome de Eagle: reporte de un caso. Odontoestomatol. 2012; 14(20): 26-31.
7. Boskey AL. Biomineralization: Conflicts, challenges, and opportunities. J Cell Biochem. 1998; 72 (30): 83-91.

8. Tísner J, Tísner B, Abad J, Abad M. Calcificación del ligamento estilohioideo: estilalgia de Aubin y síndrome de Eagle. Aportación de 5 casos clínicos. ORL ARAGON. 2003; 6 (2): 5-12.

9. Carmada AJ, Deschamps C, Forest D. Stylohyoid chain ossification: A discussion of etiology. Oral Surg Oral Med Oral Pathol. 1989, 67:508-14.

10. Langlais R, Miles D, Van Dis M. Elongated and mineralized stylohyoid ligament complex: A proposed classification and report of a case of Eagle's síndrome. Oral Surg Oral Med Oral Pathol. 1986; 61(5):527-32.

11. Khojastepour L, Dastan F, Ezoddini-Ardakani F. Evaluation of the Elongation and Calcification Patterns. Dentomaxillofac Radiol. 2015; 3 (4): 26-31.

12. Abdel-Ghany A, Osman N. Role of three-dimensional multidetector computerized tomography in diagnosis of Eagle's syndrome. The Egyptian Journal of Radiology and Nuclear Medicine. 2014; 45: 105-8.

13. Sudhakara R, Sai C, Sai N, Maghavendra M, Satish A. Prevalence of elongation and calcification patterns of elongated styloid process in South India. J Clin Exp Dent. 2013; 5(1): 30-5.

14. Öztunç H, Evlice B, Tatli U, Evlice A. Cone-beam computed tomographic evaluation of styloid process: a retrospective study of 208 patients with orofacial pain. Head \& Face Medicine. 2014; 10(5): 1-7. DOI: https://doi.org/10.1186/1746-160X-10-5

15. Basekim C, Mutlu H, Güngör A, et al. Evaluation of styloid process by three-dimensional computed tomography. Eur Radiol. 2005; 15 (1): 134-9. 
16. Akar G, Alpoz E, Govsa F, Lomcali G. Does the state of dentition effect the type of stylohyoid chain calcification pattern? Surg Radiol Anat. 2016;38(7):817-23. doi: 10.1007/s00276-016-1624-y

17. Andrei F, Motoc A, Didilescu A, Rusu M. A 3D cone beam computed tomography study of the styloid process of the temporal bone. Folia Morphol. 2013; 72 (1): 29-36.

18. Marçal E, Aguirre O, De Morais S, De Musis C, Andrade P, Henrique A. Prevalence of Elongated Styloid Process in a Central Brazilian Population. J Clin Diagn Res. 2015; 9 (9): 90-2.

19. Bagga M, Kumar C, Yeluri G. Clinicoradiologic evaluation of styloid process calcification. Imaging Sci Dent. 2012; 42: 155-6.

20. Paula M, Carraretto F. Prevalência do alongamento do proceso estilóide em pacientes portadores de desordens temporomandibulares. Rev Imagem. 2008; 30(1):1-5.

21. Anbiaee N, Javadzadeh A. Elongated styloid process: Is it a pathologic condition?. Indian J Dent Res. 2011; 22(5): 673-7. 